\title{
Clinical, radiological and pathological features of ABCA3 mutations in children
}

\author{
M L Doan, ${ }^{1}$ R P Guillerman, ${ }^{2}$ M K Dishop, ${ }^{3}$ L M Nogee, ${ }^{4}$ C Langston, ${ }^{3}$ G B Mallory, \\ M M Sockrider, ${ }^{1} \mathrm{~L} L$ Fan $^{1}$
}

\section{See editorial, p 295}

${ }^{1}$ Pediatric Pulmonary Section, Department of Pediatrics, Baylor College of Medicine, Texas Children's Hospital, Houston, Texas, USA; ${ }^{2}$ Department of Radiology, Baylor College of Medicine, Texas Children's Hospital, Houston, Texas, USA; ${ }^{3}$ Department of Pathology, Baylor College of Medicine, Texas Children's Hospital, Houston, Texas, USA; ${ }^{4}$ Division of Neonatology, Department of Pediatrics, Johns Hopkins University School of Medicine, Baltimore, Maryland, USA

Correspondence to: Dr L L Fan, Texas Children's Hospital, 6621 Fannin, CC1040. 00, Houston, TX 77030, USA: Ilfan@texaschildrenshospital.org

Received 4 May 2007 Accepted 17 October 2007 Published Online First 16 November 2007

\begin{abstract}
Background: Mutations in the $\mathrm{ABCA} 3$ gene can result in fatal surfactant deficiency in term newborn infants and chronic interstitial lung disease in older children. Previous studies on ABCA3 mutations have focused primarily on the genetic abnormalities and reported limited clinical information about the resultant disease. A study was undertaken to analyse systematically the clinical presentation, pulmonary function, diagnostic imaging, pathological features and outcomes of children with ABCA3 mutations.
\end{abstract}

Methods: The records of nine children with $\mathrm{ABCA} 3$ mutations evaluated at Texas Children's Hospital between 1992 and 2005 were reviewed and their current clinical status updated. Previous diagnostic imaging studies and lung biopsy specimens were re-examined. The results of DNA analyses were confirmed.

Results: Age at symptom onset ranged from birth to 4 years. Cough, crackles, failure to thrive and clubbing were frequent findings. Mean lung function was low but tended to remain static. CT scans commonly revealed ground-glass opacification, septal thickening, parenchymal cysts and pectus excavatum. Histopathological patterns included pulmonary alveolar proteinosis, desquamative interstitial pneumonitis and non-specific interstitial pneumonitis, and varied with age. Dense abnormalities of lamellar bodies, characteristic of $\mathrm{ABCA} 3$ mutations, were seen by electron microscopy in all adequate specimens. Outcomes varied with the age at which the severity of lung disease warranted open lung biopsy, and some patients have had prolonged survival without lung transplantation.

Conclusions: The presentation and course of interstitial lung disease due to $\mathrm{ABCA} 3$ mutations are variable, and open lung biopsy and genetic testing are warranted early in the evaluation of children with a consistent clinical picture.

An important development in the field of childhood interstitial lung disease (chILD) has been the discovery of inborn errors of surfactant metabolism, including mutations in the surfactant protein B (SP$\mathrm{B})$, surfactant protein $\mathrm{C}$ (SP-C) and $\mathrm{ABCA} 3$ genes. ${ }^{1-3}$ Mutations in these genes cause chILD with varying histopathological patterns including desquamative interstitial pneumonitis (DIP), chronic pneumonitis of infancy, pulmonary alveolar proteinosis (PAP) and non-specific interstitial pneumonitis (NSIP). ${ }^{1-10}$ ABCA3 is an ATP-binding cassette transporter of lipids that is found in the limiting membrane of lamellar bodies in alveolar type II cells. ${ }^{11}$ Initially reported as a cause of fatal lung disease in term neonates with a clinical picture similar to that of SP$\mathrm{B}$ deficiency, ${ }^{3}$ mutations in the $\mathrm{ABCA} 3$ gene are now known also to cause chronic interstitial lung disease (ILD) in older patients. ${ }^{8}$ Previous reports on ABCA3 mutations focused primarily on the genetic abnormalities and gave limited clinical information about the resultant disease beyond the neonatal period. We present an analysis of nine children with ABCA3 mutations evaluated at a single medical centre in order to describe more fully the clinical presentations, pulmonary function, diagnostic imaging, pathological features and outcomes of children with this disorder.

\section{METHODS}

Nine children who were eventually diagnosed with mutations in the ABCA3 gene were evaluated at Texas Children's Hospital (TCH), a tertiary chILD and pediatric lung transplantation referral centre, between 1992 and 2005. Five of the patients were transferred to or primarily cared for at our centre while four were seen on an outpatient referral basis. The records of patients were reviewed to document the neonatal history, interval history prior to initial evaluation at $\mathrm{TCH}$, presenting symptoms, family and environmental history, physical findings at initial evaluation, pulmonary function tests and treatment regimen. Each patient's current clinical status-including growth parameters, pulmonary function and ILD score $\mathrm{s}^{12}$ was obtained from clinical records and communications with parents and referring physicians. Records of the initial evaluation were available and adequate for analysis in each case, and the clinical status was updated for all survivors. All available chest radiographs, CT scans and lung pathology specimens were systematically re-examined (without blinding) by a paediatric radiologist (RPG) and a paediatric lung pathologist (MKD), respectively, at TCH. DNA samples from seven patients were initially analysed for ABCA3 mutations by direct sequence analysis of the 30 coding exons as previously described under a research protocol at the Johns Hopkins University School of Medicine, and their mutations were subsequently confirmed in the CLIA certified Johns Hopkins DNA Diagnostic Laboratory where the mutations of the remaining two patients were also identified. Two patients ( 1 and 6 ) have been described in previous publications. $^{8} 13$

\section{RESULTS}

\section{Clinical presentation}

All nine patients in our cohort were born at term. Five had respiratory symptoms in the newborn period, including three patients who developed 
Table 1 Characteristics of nine children with ABCA3 mutations

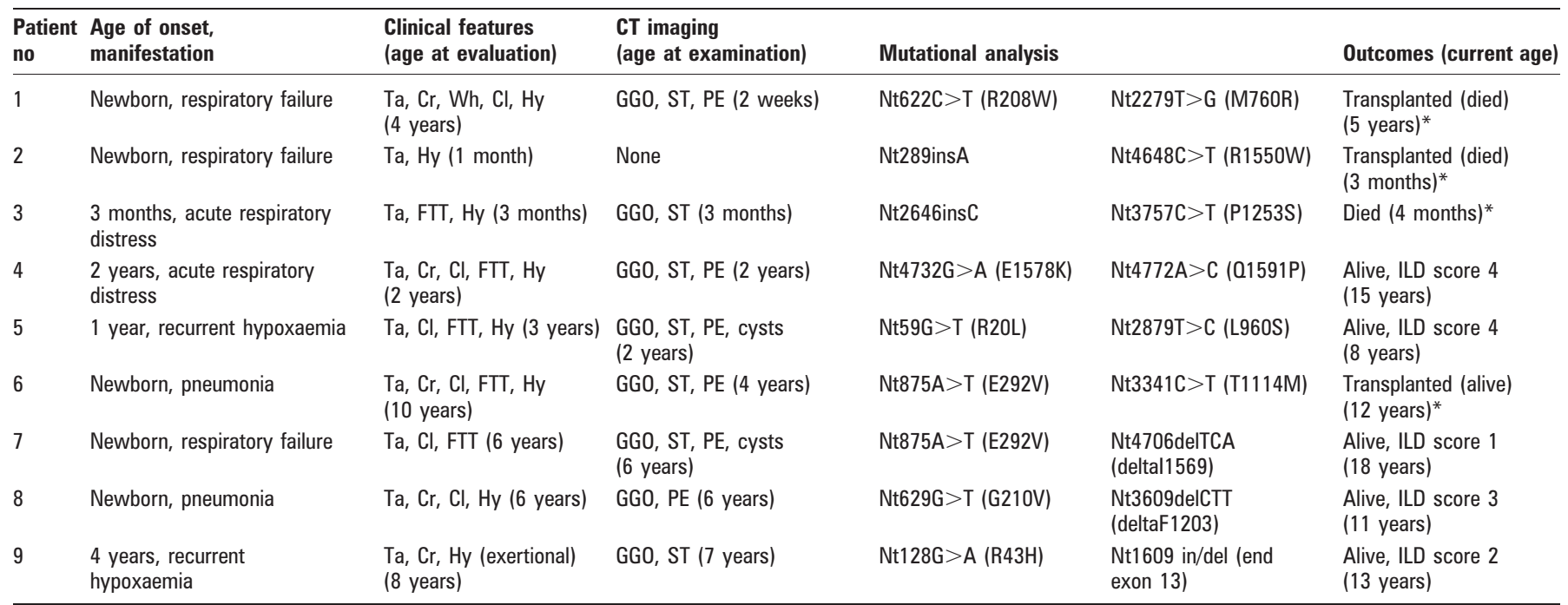

Ta, tachypnoea; $\mathrm{Cr}$, crackles; Wh, wheezing; $\mathrm{Cl}$, clubbing; Hy, hypoxaemia; FTT, failure to thrive; GG0, ground-glass opacification; ST, septal thickening; PE, pectus excavatum. ${ }^{*}$ Age at transplantation or death.

respiratory failure (two were extubated after 2 days; one had progressive deterioration) and two patients who were treated for pneumonia with intravenous antibiotics and/or oxygen. In the four patients who were eventually discharged home, three continued to have either persistent or intermittent respiratory symptoms (described below), while one infant was apparently
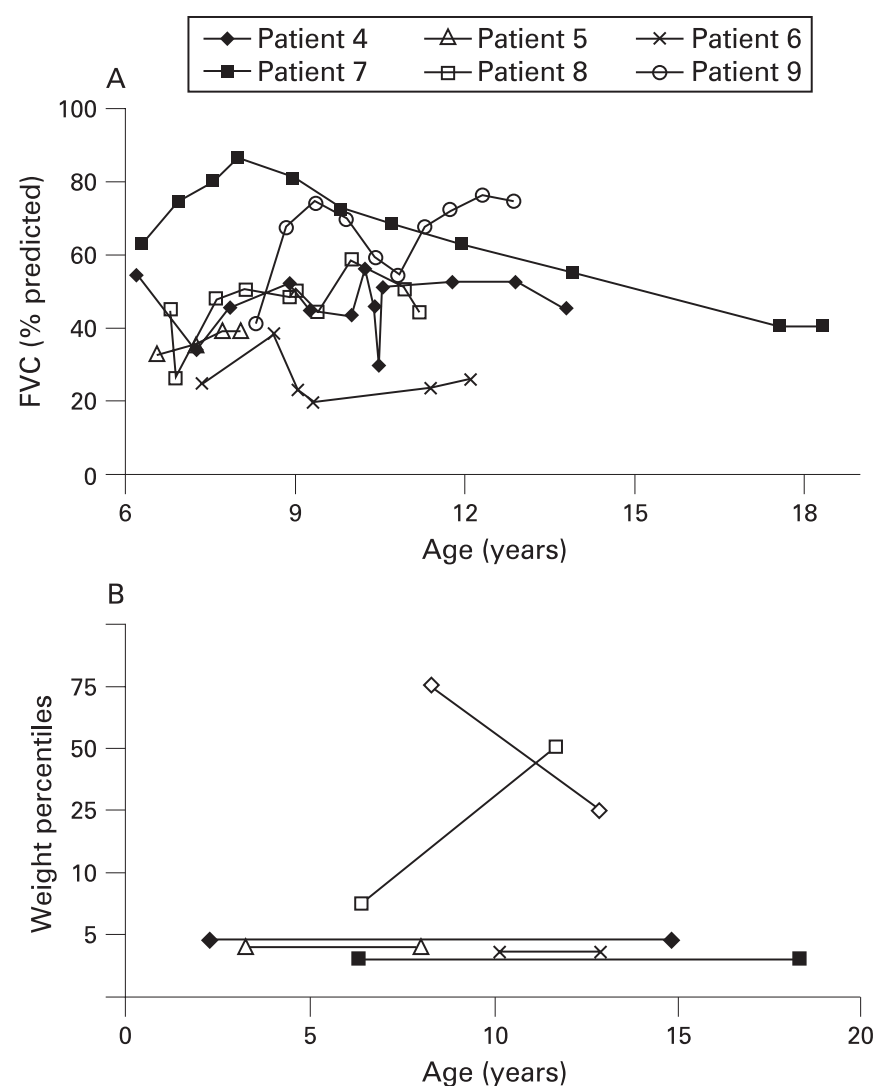

Figure 1 (A) Changes in forced vital capacity (FVC) percentage predicted over time in six patients with $A B C A 3$ mutations. (B) Changes in weight percentile in the same six patients plotted on a representation of the standard growth curve. Data points in the bottom bar simply represent $<5$ th percentile. well until 8 months of age. In the other four children who had an unremarkable neonatal course, the age at symptom onset ranged from 3 months to 4 years. Known environmental exposures were present in three patients: one to birds and two to cigarette smoke. A family history of chILD was absent at the time of initial evaluation in all nine cases.

The eight patients who did not have persistent respiratory failure from the newborn period all presented later with cough, tachypnoea, dyspnoea and exercise intolerance (table 1). Most had hypoxaemia at rest, while one had hypoxaemia only with exertion. On initial evaluation at our centre, the majority of patients were below the 5th percentile for weight and had crackles and retractions on examination. Only one child had wheezing. The seven children who were older than 2 years at initial evaluation all had clubbing, and five of them also had pectus excavatum apparent on physical examination.

\section{Pulmonary function tests}

Six patients were able to perform spirometry starting at age 68 years (fig 1A). The mean (SD) initial forced vital capacity (FVC) and forced expiratory volume in $1 \mathrm{~s}\left(\mathrm{FEV}_{1}\right)$ percentage predicted were $43.6(13.9) \%$ and $41.2(12.0) \%$, respectively, consistent with severe restrictive lung disease. Most of the patients were unable to perform the manoeuvre for measurement of the carbon monoxide lung transfer factor (TLCO).

\section{Diagnostic imaging}

Chest radiographs were available for review for all nine patients at various ages. They consistently showed bilateral diffuse or patchy hazy granular or streaky opacities (fig 2). One or more helical or high-resolution axial CT (HRCT) scans were available for review in eight patients; the ninth was too critically ill to undergo CT imaging. On CT scan, all patients had ground-glass opacification that was either diffuse throughout the lungs or patchy but involving multiple or all lobes. The imaging appearance during infancy is very similar to neonatal respiratory distress syndrome. In some patients the intensity of the groundglass opacification decreased with age, but this did not correlate with improvement in lung function or degree of hypoxaemia. All patients imaged beyond infancy developed fine or coarse peripheral interstitial septal thickening. Five patients eventually 
Figure 2 Major radiographic patterns seen with $A B C A 3$ mutations. (A) Anteroposterior chest radiograph from a 3-month-old girl showing diffuse hazy granular pulmonary opacification. (B) Axial high-resolution CT (HRCT) image of the same infant showing diffuse groundglass opacification of the lungs. (C) Axial HRCT image from a 6-year-old girl showing mosaic lung attenuation with widespread ground-glass opacification. (D) Imaging of the same child at 9 years of age showing continued widespread ground-glass attenuation in a similar distribution and development of small airfilled lung cysts and thickening of the peripheral pulmonary interstitium.
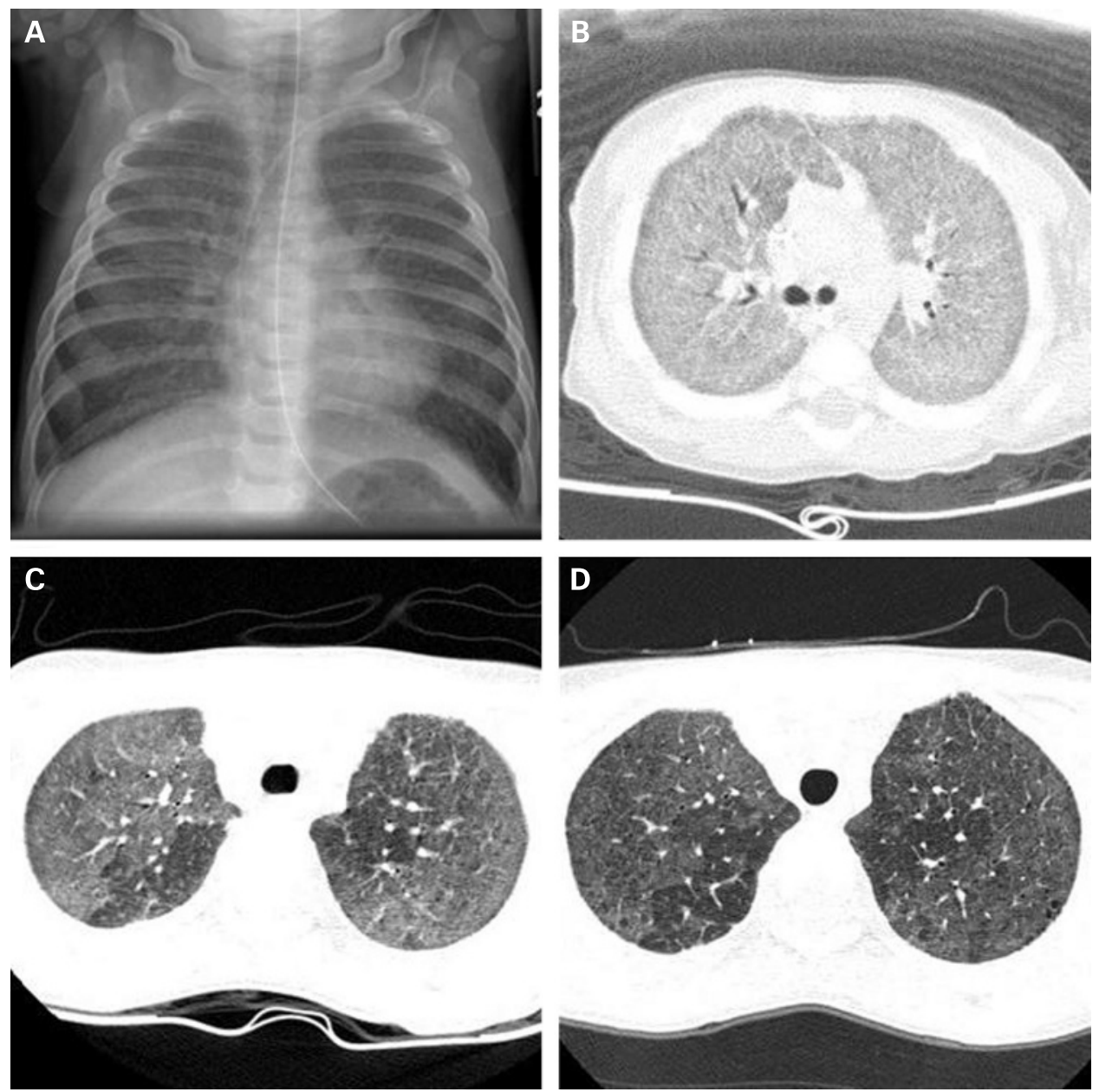

developed small (few $\mathrm{mm}$ in diameter) air-filled parenchymal lung cysts that tended to increase in number and size over time. Hilar and mediastinal lymphadenopathy (four patients), consolidation and atelectasis that cleared over time (four patients), pleural thickening (three patients) and air trapping (three patients) were also seen. Pectus excavatum (defined as Haller index $>2.7)^{14}$ developed in all patients surviving beyond infancy.

\section{Pathology}

Diagnostic lung biopsy slides were available for review in all nine patients, including three with a second biopsy and two with subsequent lung pathology specimens after transplantation and at autopsy. The time interval between specimens was $<1$ month for three patients and similar histological features were seen in the paired specimens in each case. Two subjects (patients 1 and 6) had an extended interval between biopsies (4 years in both cases), and these biopsies are considered separately. Histological features and major patterns of disease for these 11 biopsies are summarised in table 2 . The spectrum of overlapping histological findings were grouped into three major categories: PAP pattern, DIP pattern and NSIP pattern (fig 3). Features present in all 11 cases included some degree of lobular remodelling, at least focally increased alveolar macrophages and at least focal pneumocyte hyperplasia. All cases showed either alveolar proteinosis material or cholesterol clefts (endogenous lipoid pneumonia), with both features present in five biopsies. Alveolar proteinosis material and diffuse alveolar epithelial hyperplasia were most prominent in the infants and were inconspicuous in the older children at the time of biopsy. Ironladen macrophages were present in all patients except for the two youngest (aged 3 weeks and 1 month). Lobular remodelling was most prominent in the older children, although this finding was variable with some biopsies showing a patchy distribution of architectural abnormalities.

The two patients with follow-up biopsies at prolonged intervals allowed for examination of the progression of histological findings over time. When a biopsy specimen was taken at 3 weeks of age, patient 1 initially had a PAP pattern with early lobular remodelling and a reactive widened interstitium (fig 4A and B). At follow-up biopsy 4 years later, the lung showed more extensive lobular remodelling (airspace enlargement and increased interstitial smooth muscle), mildly increased interstitial inflammatory cells, but less reactive interstitial widening (fig 4C and D). Alveolar proteinosis was present in both biopsies, with the additional finding of endogenous lipoid pneumonia at 4 years of age. Patient 6 underwent biopsies at 4 years (fig 4E) and 9 years of age (fig 4F), both showing an NSIP pattern with endogenous lipoid pneumonia and extensive lobular remodelling. Both specimens showed increased lymphoid infiltrates and cholesterol clefts, although these findings were more prominent in the second biopsy.

Tissue was available for electron microscopic study in six biopsies, with adequate preservation of the ultrastructure in five cases. Characteristic small lamellar dense bodies were identified in all five patients but were more frequent and well formed in 


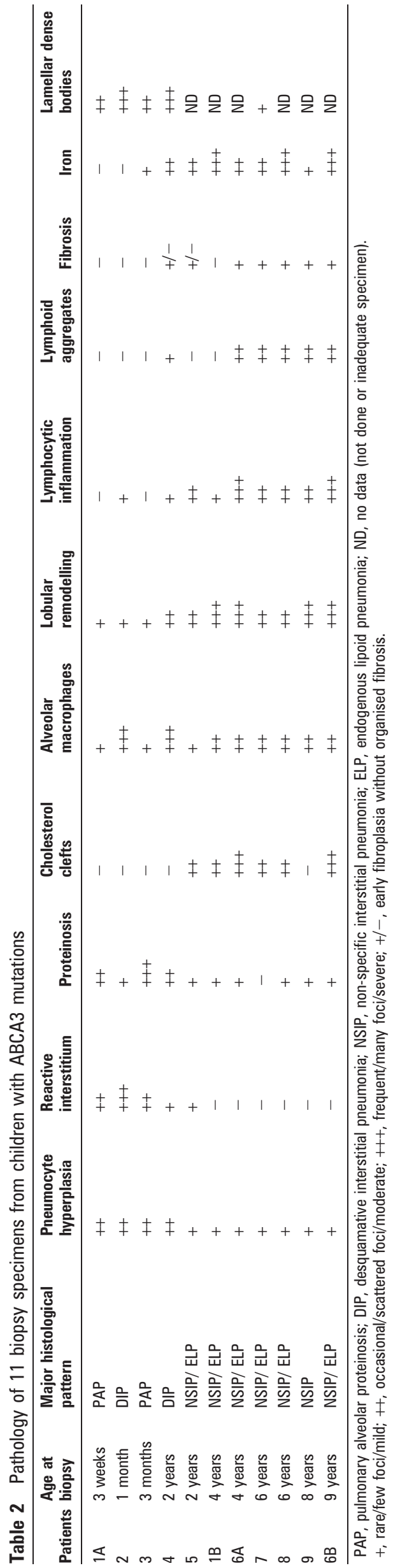

the younger children (age range 3 weeks to 2 years) and inconspicuous in the one biopsy from an older child (age 6 years) (fig 5). The dense bodies varied in size and location, including both centrally positioned and eccentric round electron-dense forms. The lamellar structure associated with the dense bodies was delicate and whorled in some areas and more compact with increased electron density in other areas. In addition to these dense bodies, the type II alveolar epithelial cells also showed increased lysosomal material, forming dense black globular cytoplasmic aggregates focally containing components of lamellar membranes, suggesting intralysosomal degeneration of abortive lamellar bodies. Composite lamellar bodies with apparent fusion of two or three whorled lamellar bodies were also detected at least focally in each case. The abnormal dense bodies and degenerating lysosomal lamellar structures were also admixed with a few lamellar bodies of normal size and structure in each case, and these normal lamellar forms predominated in the oldest child (age 6 years).

\section{Genetics}

There were eight Caucasians and one Hispanic in the cohort. DNA analysis for mutations in the ABCA3 gene revealed two mutations in all nine patients consistent with autosomal recessive inheritance. Five of these mutations (R20L, $\Delta$ F1203, E292V, T114M, Q1591P) have been previously identified in subjects with chILD, whereas the remainder are novel. The E292V mutation was the only one found in more than one patient. Eight patients had been followed for many years with a diagnosis of idiopathic chILD, while one (patient 1) was prospectively diagnosed at 2 months of age.

\section{Treatment and outcome}

All nine patients were treated with systemic corticosteroids, seven with pulses of intravenous methylprednisolone given on a monthly basis for varying durations. Hydroxychloroquine was added to the treatment regimen of seven patients. Records were not sufficiently complete to determine the short-term response to treatment. Long-term outcomes within our cohort were variable. One infant died from unrelenting respiratory failure at 4 months of age, 1 month after initial presentation (table 1). Three patients underwent lung transplantation at ages 3 months, 5 years and 12 years; the youngest was the child with persistent respiratory failure from the neonatal period. The two youngest transplant recipients have died (one from primary graft dysfunction and one from bronchiolitis obliterans), while the oldest is still alive $<1$ year after transplantation.

The five survivors who have not required transplantation currently range in age from 8 to 18 years. Three of them have had an improvement in the degree of their hypoxaemia (as evaluated by ILD score) while two have not. None have evidence of secondary pulmonary arterial hypertension by electrocardiography or echocardiography. The lung function in four of these five patients has not changed significantly over time; the fifth has had a slow decline from a peak $\mathrm{FEV}_{1}$ of $80 \%$ to $40 \%$ predicted over 10 years, although he currently reports no symptoms and is very active physically. The mean (SD) latest FVC and $\mathrm{FEV}_{1}$ were 48.9 (14.8)\% predicted and 48.1 (18.5)\% predicted, respectively, in the five survivors without transplant. In the six patients who are still alive, all four who had a weight $<5$ th percentile at the initial evaluation are still at $<3$ rd percentile for weight, while the two children who did not have failure to thrive have continued to have normal growth with follow-up intervals ranging from 2 to 12 years (fig 1B). 

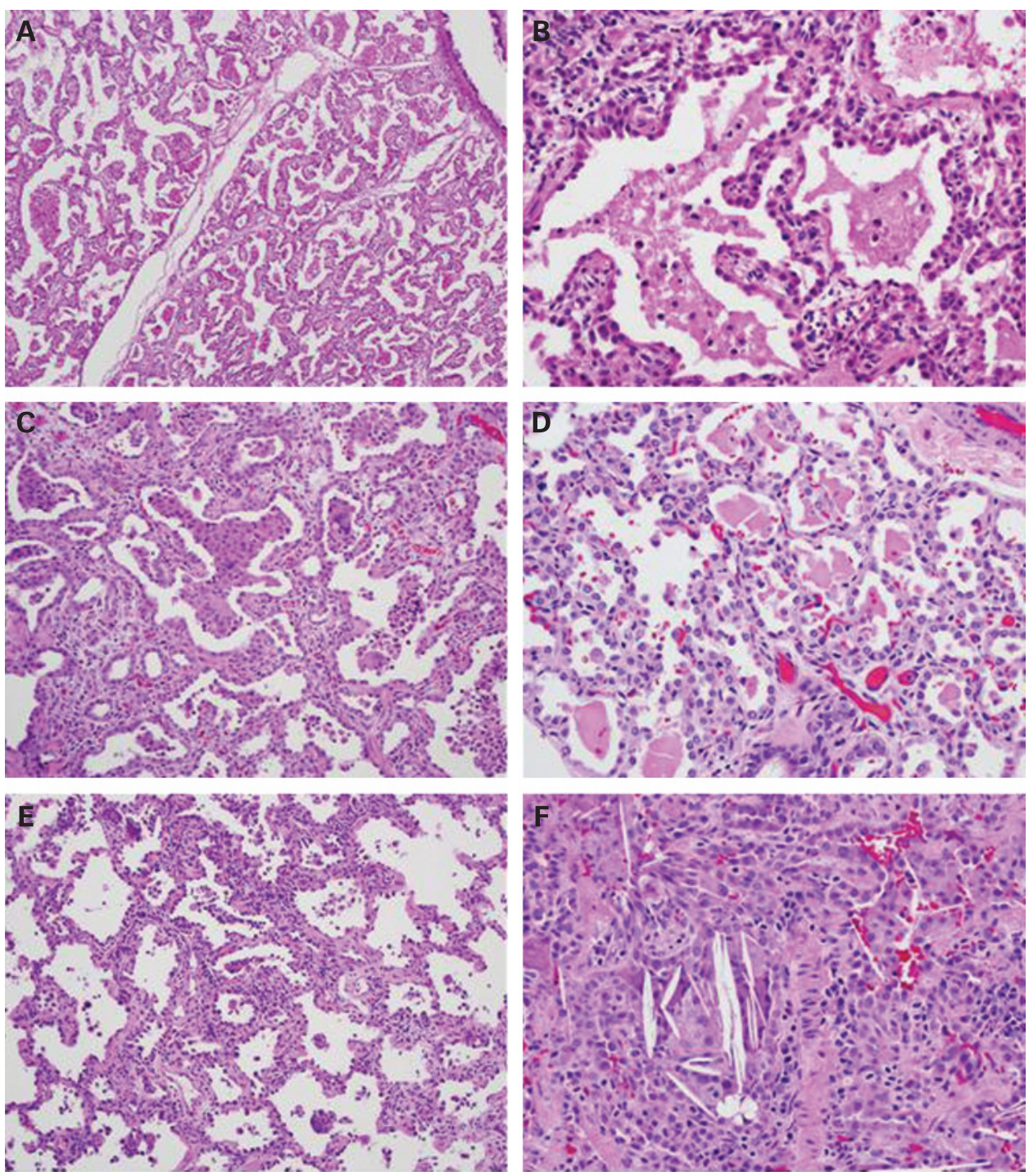

Figure 3 Major histological patterns associated with ABCA3 mutations. (A, B) Pulmonary alveolar proteinosis (PAP) pattern in infancy is characterised by early lobular remodelling, interstitial widening, diffuse alveolar epithelial hyperplasia and predominantly fine granular proteinosis material admixed with foamy macrophages. There are no significant inflammatory infiltrates. (A, B, 3-month-old girl, $4 \times$ and $20 \times$ original magnification, respectively). (C, D) Desquamative interstitial pneumonitis (DIP) pattern in infancy is characterised by prominent clustered alveolar macrophages (C, 2month-old girl, $10 \times$ original magnification). Other findings may include interstitial widening, diffuse reactive alveolar epithelial hyperplasia and focal globular proteinaceous material (D, 2-month-old girl, 20× original magnification). Inflammatory infiltrates are inconspicuous. (E, F) Non-specific interstitial pneumonitis (NSIP) pattern in older children is characterised by lobular remodelling, increased interstitial smooth muscle and patchy mild interstitial lymphocyte infiltrates ( $E$, 6-year-old girl, $10 \times$ original magnification). These findings are often associated with cholesterol granulomas (endogenous lipoid pneumonia), with or without lymphoid hyperplasia (F, 2-year-old boy, 20× original magnification).

Within our cohort no specific clinical feature correlated with outcome, as measured by the need for lung transplantation or death, although these analyses were difficult because of the small number of patients. There was a suggestion that younger age at the time of initial lung biopsy might indicate a lower likelihood of surviving without transplantation (fig 6).

\section{DISCUSSION}

Our study is the first to present a systematic review of the clinical features, lung function, imaging changes and histopathology of children with $\mathrm{ABCA} 3$ mutations. The results of our analysis extend the descriptions of previous studies and reveal several clinically significant findings not previously described.

Although $\mathrm{ABCA} 3$ mutations are inherited in an autosomal recessive fashion, ${ }^{3}$ none of our patients had a positive family history at the time of presentation, underscoring the need to consider this entity even in the absence of such history. The higher proportion of cases with a positive family history reported in previous studies may reflect selected patient populations..$^{3810}$ The age at symptom onset for nearly half of our patients was beyond the neonatal period and extended to early childhood, re-emphasising the findings of Bullard et al ${ }^{\mathrm{B}}$ that ABCA3 mutations can present as chronic ILD in young children.

This study provides the first description of the serial lung function, growth and radiographic changes in children with ABCA3 mutations. The FVC percentage predicted, while initially low, tended to remain stable over many years. Among the survivors without lung transplantation, the majority had a mild improvement in oxygenation. Most of the children who presented with chronic ILD beyond the 

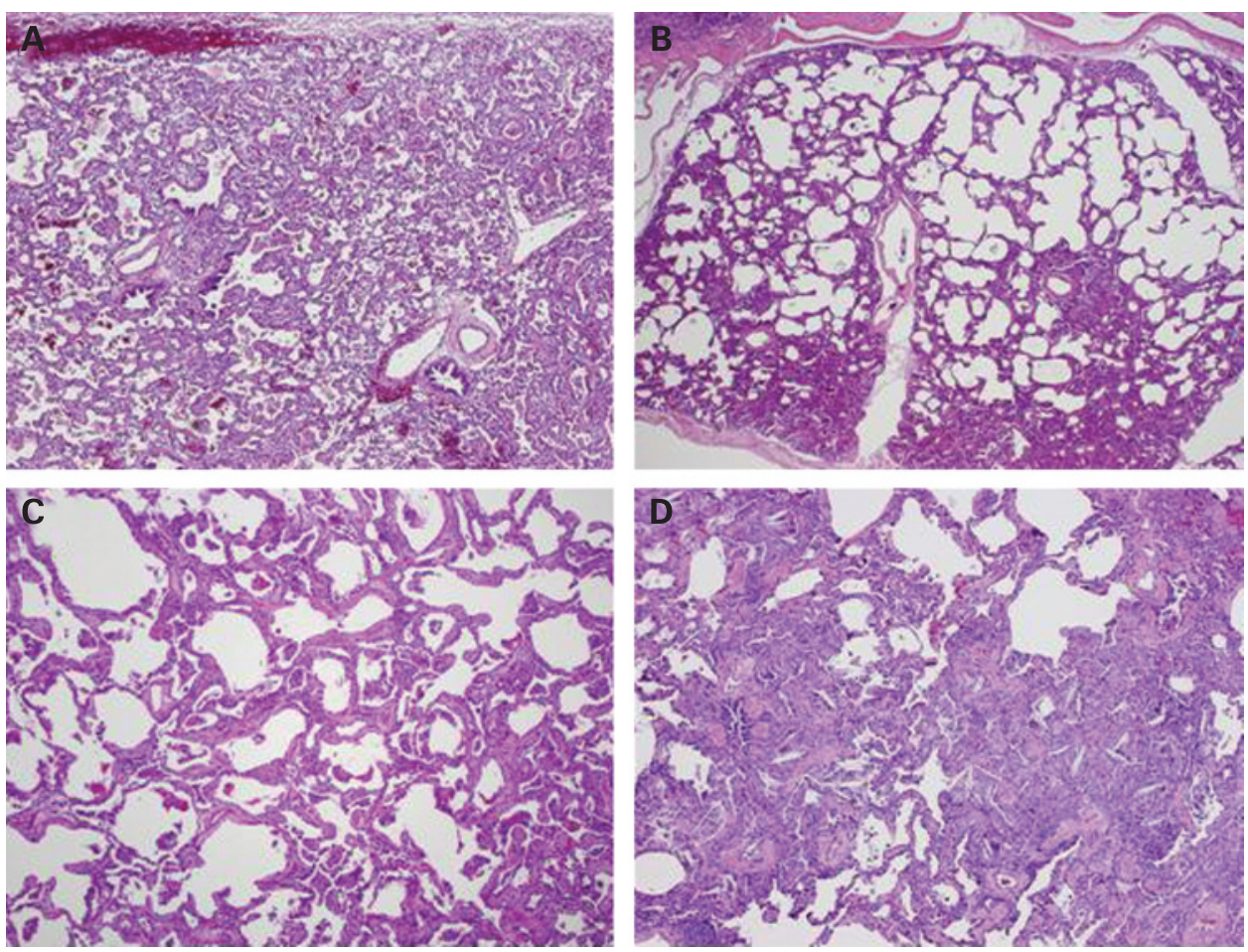

Figure 4 Progression of disease in follow-up biopsies. (A, B) Patient 1 initially had a pattern of variant pulmonary alveolar proteinosis (PAP) at 3 weeks of age (A), followed by a predominant pattern of non-specific interstitial pneumonitis (NSIP) at 4 years of age (B). Compared with the first biopsy, the second shows increased airspace enlargement with more pronounced lobular remodelling and interstitial smooth muscle, decreased interstitial widening, similar granular and globular proteinosis material, and occasional foci of endogenous lipoid pneumonia $(A, 4 \times$ original magnification; B, $2 \times$ original magnification). (C, D) Two biopsies in patient 6 both show significant lobular remodelling and increased interstitial smooth muscle, as well as clusters of foamy macrophages. The biopsy at 4 years of age shows an NSIP pattern with patchy mild interstitial lymphocyte infiltrates and only focal endogenous lipoid pneumonia (C, $4 \times$ original magnification). The follow-up biopsy at 9 years of age also shows an NSIP pattern, but with much more prominent lymphocytic infiltrates and frequent cholesterol clefts $(\mathrm{D}, 4 \times$ original magnification).
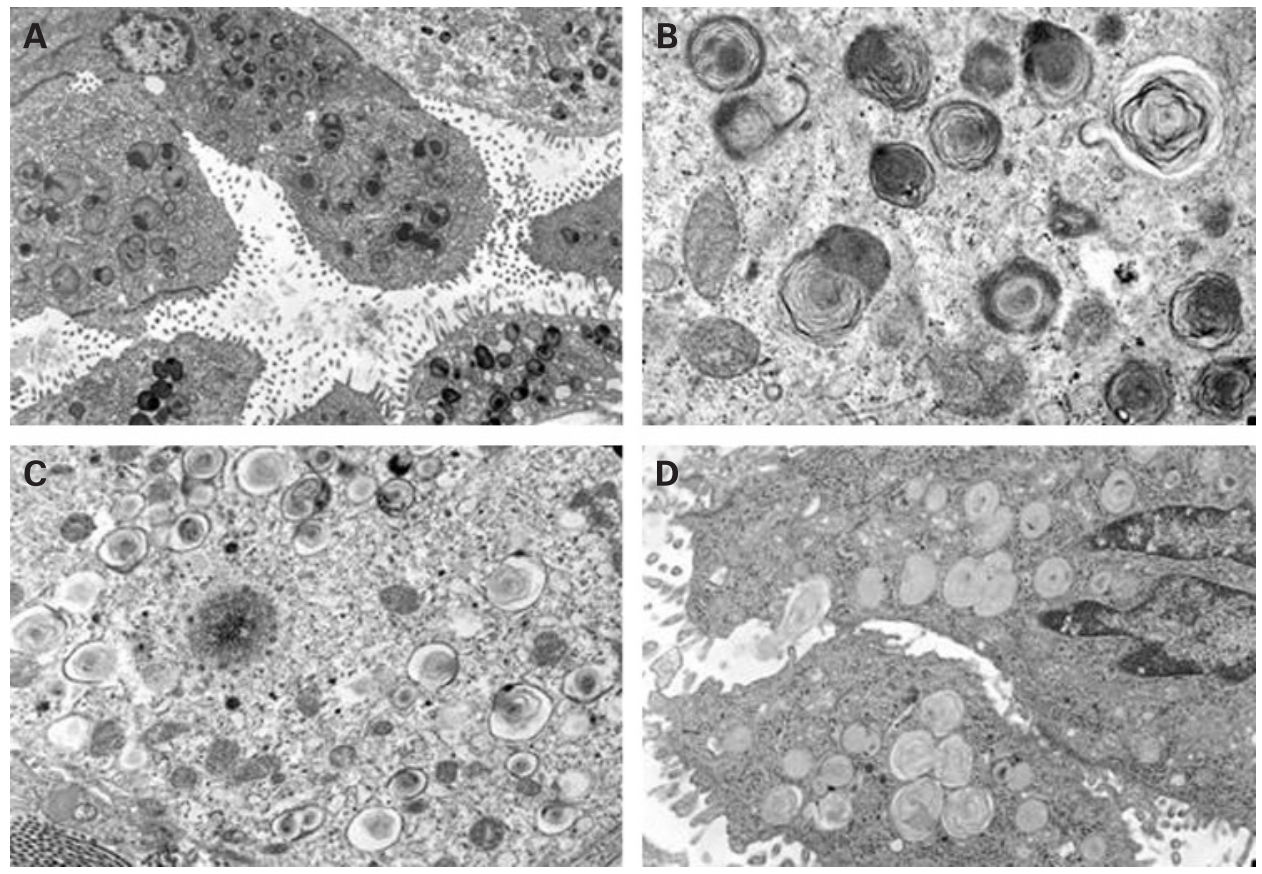

Figure 5 Electron microscopic features of ABCA3 mutations. (A, B) Type II alveolar epithelial cells contain characteristic abnormal lamellar bodies with distinctive central and eccentric round dense bodies (A, 2-month-old girl, $4000 \times$ original magnification; B, 2-year-old boy, $25000 \times$ original magnification). A few degenerating forms suggesting lysosomal degradation are also noted in some cases. Many cases also contain a few normal lamellar bodies ( $B$, upper right) admixed with the abnormal forms. (C) Findings in a 6-year-old boy include only rare characteristic dense bodies with many lamellar bodies that are small with vague irregular densities (C, $12000 \times$ original magnification) while others show normal size with appropriate delicate whorled structure (D, $10000 \times$ original magnification). Occasional fusion of lamellar bodies is also noted (D). 


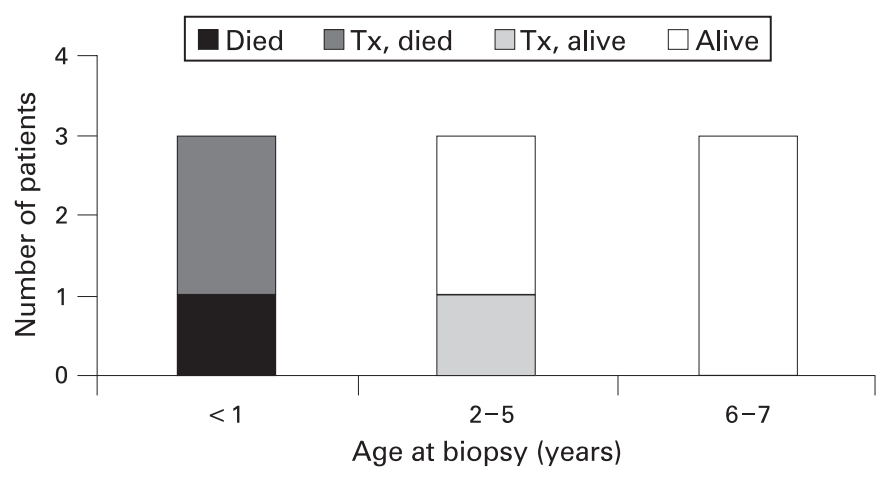

Figure 6 Outcomes in the nine patients with ABCA3 mutations in relation to age at open lung biopsy. Tx, transplant.

neonatal period had failure to thrive, and all of these patients continued to grow poorly. The remainder, who underwent open lung biopsy at older ages (suggesting milder disease), did not have growth abnormalities at initial evaluation and they continued to grow well. Serial CT imaging of the lungs consistently showed bilateral ground-glass opacification that in some cases decreased in intensity with age, while peripheral interstitial thickening and small parenchymal lung cysts became more prominent findings over time. Interestingly, pectus excavatum was seen in all patients who were imaged beyond infancy; one child underwent surgical correction of his pectus before he was diagnosed with ABCA3 mutations. We are aware of only one previous report of pectus excavatum in association with chronic ILD in a patient with pulmonary alveolar microlithiasis. ${ }^{15}$ Changes in CT findings over time did not correlate with lung function, hypoxaemia or outcome, suggesting that once the diagnosis is established, frequent routine imaging studies are not warranted.

The systematic review of all open lung biopsy specimens in our series-including repeat biopsies at older ages in two children-showed that the pathological phenotypes of ABCA3 mutations are age-dependent. In the biopsy specimens obtained from young infants, PAP and DIP were the predominant patterns. In the two biopsy specimens from toddlers, one showed a DIP pattern and the other had an NSIP pattern. Finally, the older children-who underwent biopsy at 4-9 years of age-all had an NSIP pattern, often with superimposed endogenous lipoid pneumonia. No cases showed a complete constellation of features seen in chronic pneumonitis of infancy. As this pattern is most often recognised in older infants (618 months) with SP-C mutations, its absence in our series may reflect this gap in age distribution. While different histological patterns have been reported in previous studies of ABCA3 mutations, ${ }^{3-10}$ an age-dependent variation in such patterns has not been described. Together with earlier reports, our findings emphasise that disease-causing mutations in the ABCA3 gene can lead to multiple pathological diagnoses. It is important to note that several of our patients had been followed for many years (in one case into adulthood) with the pathological diagnosis of NSIP or DIP, raising the possibility that some adults with long-standing chronic ILD who have these pathological patterns may actually have ABCA3 mutations.

The presence of characteristic small lamellar dense bodies in all five patients in our cohort who had adequately preserved samples for electron microscopic examination suggests that this finding may be a sensitive diagnostic feature of ABCA3 mutations. The absence of mature lamellar bodies and accumulation of these central or eccentrically placed dense bodies have previously been reported in patients with ABCA3 mutations. ${ }^{3} 10^{13} 1^{16-18}$ In three of our five patients some normal lamellar bodies were also seen in type II alveolar cells, which previously had been described in only one patient. ${ }^{10}$ This finding further underscores the variability in the histology that can occur with ABCA3 mutations. More importantly, however, given that results of DNA analyses are not quickly available, emphasis must be placed on the need to preserve a portion of any open lung biopsy done for evaluation of chILD for electron microscopy analysis, as it may provide a rapid preliminary diagnosis of $\mathrm{ABCA} 3$ mutations. Guidelines for the processing of paediatric lung biopsies have recently been published. ${ }^{19}$

The significant heterogeneity and number of novel mutations in the ABCA3 gene found in our patients is similar to the report by Brasch et $a l^{10}$ and suggests that, like other genetic conditions (eg, cystic fibrosis), many more mutations may yet be discovered. The E292V mutation found in two of our patients has been reported as the most common defect in the ABCA3 gene leading to chILD. ${ }^{20}$ Our study shows that the outcome in children with ABCA3 mutations is also quite variable. Almost half of our patients have survived into the second decade of life without need for lung transplantation. However, we realise that a selection bias may exist in our study due to the referral pattern.

The clinical and radiological features described above for $\mathrm{ABCA} 3$ mutations can overlap with those seen with other forms of inborn errors of surfactant metabolism (SP-B and SP-C mutations). Respiratory distress syndrome in term newborns can result from either SP-B or ABCA3 mutations, ${ }^{1}$ while chronic ILD starting in infancy or early childhood can be the manifestation of either SP-C or ABCA3 mutations. ${ }^{2} 45721-24$ Young children with ILD due to SP-C mutations can have similar findings on $\mathrm{CT}$ imaging as those with $\mathrm{ABCA} 3$ mutations. ${ }^{72} 22$ While histological findings can also overlap, ${ }^{147} 25$ analysis of the lung ultrastructure using electron microscopy may be able to distinguish between these three entities. Patients with SP-B mutations have large, membrane-bound, pleomorphic, cytoplasmic inclusions with irregularly disposed lamellae and multiple associated vesicular structures, ${ }^{17}$ while patients with SP-C mutations can have normal or (less commonly) somewhat disorganised-appearing lamellar bodies. ${ }^{4} 2324$

Based on the results of our analysis, we conclude that ABCA3 mutations should be considered in the differential diagnoses of newborns with unexplained respiratory distress syndrome and for older infants and young children who have chronic ILD of unclear aetiology. Findings on HRCT scanning-particularly widespread ground-glass opacification, septal thickening and parenchymal lung cysts - can provide supportive evidence of a possible inborn error in surfactant metabolism. DNA analysis for mutations in the $\mathrm{ABCA} 3$ gene is necessary for a definitive diagnosis and should also be considered for the SP-B and SP-C genes because of similarities in the clinical, radiological and pathological features of these entities. In patients with suspected ABCA3 mutations and chronic stable disease, confirmatory genetic testing will eliminate the need for a lung biopsy. If genetic testing is equivocal or non-diagnostic, a lung biopsy specimen should be obtained to look for one of the compatible histological patterns and to evaluate for characteristic lamellar dense bodies by electron microscopy. A lung biopsy should also be considered in patients with suspected ABCA3 mutations and rapidly progressive disease. These patients could be candidates for lung transplantation, and awaiting genetic testing results would delay a timely diagnosis. 
Outcomes in patients with ABCA3 mutations are variable, ranging from severe irreversible respiratory failure in early infancy to chronic static or progressive interstitial lung disease, with many patients surviving well into their second decade without lung transplantation.

Acknowledgements: The authors thank Drs Phil Black, Fran White, Stuart Sweet, Susan Wert, Gail Deutsch, Lisa Young, Aaron Hamvas, Robert Zwerdling, Jason Fullmer and Tarak Patel for their care of these patients and their assistance in our research. We recognise Mr Jim Barrish for his technical expertise in electron microscopy. We also express our appreciation to the patients and their families for their assistance and willingness to participate in the study.

Funding: LMN was supported by an NIH grant (HL-54703).

Competing interests: None.

Ethics approval: This study was approved by the Institutional Review Board of Baylor College of Medicine.

\section{REFERENCES}

1. Nogee LM, deMello DE, Dehner LP, et al. Deficiency of pulmonary surfactant protein $\mathrm{B}$ in congenital alveolar proteinosis. N Engl J Med 1993;328:406-10.

2. Nogee LM, Dunbar $A E$, Wert SE, et al. A mutation in the surfactant protein $C$ gene associated with familial interstitial lung disease. N Engl J Med 2001;344:573-9.

3. Shulenin S, Nogee LM, Annilo T, et al. ABCA3 gene mutations in newborns with fatal surfactant deficiency. N Engl J Med 2004:350:1296-303.

4. Thomas A0, Lane K, Phillips J, et al. Heterozygosity for a surfactant protein $\mathrm{C}$ gene mutation associated with usual interstitial pneumonitis and cellular nonspecific interstitial pneumonitis in one kindred. Am J Respir Crit Care Med 2002;165:1322-8.

5. Tredano M, Griese M, Brasch F, et al. Mutation of SFTPC in infantile alveolar proteinosis with or without fibrosing lung disease. Am J Med Gen 2004;126A:18-26.

6. Cameron HS, Somaschini M, Carrera P, et al. A common mutation in the surfactant protein C gene associated with lung disease. J Pediatr 2005;146:370-5.

7. Stevens PA, Pettenazzo A, Brasch F, et al. Nonspecific interstitial pneumonia, alveolar proteinosis, and abnormal proprotein trafficking resulting from a spontaneous mutation in the surfactant protein C gene. Pediatr Res 2005;57:89-98.

8. Bullard JE, Wert SE, Whitsett JA, et al. ABCA3 mutations associated with pediatric interstitial lung disease. Am J Respir Crit Care Med 2005;172:1026-31.
9. Garmany TH, Moxley MA, White FV, et al. Surfactant composition and function in patients with ABCA3 mutations. Pediatr Res 2006;59:801-5.

10. Brasch F, Schimanski S, Muhlfeld C, et al. Alteration of the pulmonary surfactant system in full-term infants with hereditary ABCA3 deficiency. Am J Respir Crit Care Med 2006;174:571-80.

11. Mulugeta S, Gray JM, Notarfrancesco KL, et al. Identification of LBM180, a lamellar body limiting protein of alveolar type II cells, as the $\mathrm{ABC}$ transporter protein $\mathrm{ABCA}$. J Biol Chem 2002;277:22147-55.

12. Fan LL, Kozinetz CA. Factors influencing survival in children with chronic interstitial lung disease. Am J Respir Crit Care Med 1997;156:939-42.

13. Prestridge A, Wooldridge J, Deutsch G, et al. Persistent tachypnea and hypoxia in a 3-month-old term infant. J Pediatr 2006;149:702-6.

14. Lawson ML, Barnes-Eley M, Burke BL, et al. Reliability of a standardized protocol to calculate cross-sectional chest area and severity indices to evaluate pectus excavatum. J Pediatr Surg 2006:41:1219-25.

15. Hasan S, Cevat 0, Sami C, et al. Pulmonary alveolar microlithiasis with pectus excavatum. Case report. Indian J Med Sci 1994;48:199-200.

16. Cutz E, Wert SE, Nogee LM, et al. Deficiency of lamellar bodies in alveolar type II cells associated with fatal respiratory disease in a full-term infant. Am J Respir Crit Care Med 2000;161:608-14.

17. Tryka AF, Wert SE, Mazursky JE, et al. Absence of lamellar bodies with accumulation of dense bodies characterizes a novel form of congenital surfactant defect. Pediatr Dev Pathol 2000:3:335-45.

18. Edwards V, Cutz E, Viero S, et al. Ultrastructure of lamellar bodies in congenital surfactant deficiency. Ultrastruct Pathol 2005;29:503-9

19. Langston C. Patterson K, Dishop MK, et al. A protocol for the handling of tissue obtained by operative lung biopsy: recommendations of the chlLD Pathology Cooperative Group. Pediatr Dev Pathol 2006;9:173-80.

20. Nogee LM. Genetics of pediatric interstitial lung disease. Curr Opin Pediatr 2006;18:287-92.

21. Amin RS, Wert SE, Baughman RP, et al. Surfactant protein deficiency in familia interstitial lung disease. J Pediatr 2001;139:85-92.

22. Soraisham AS, Tierney AJ, Amin HJ. Neonatal respiratory failure associated with mutation in the surfactant protein $C$ gene. J Perinatol 2006;26:67-70.

23. Brasch F, Griese $\mathbf{M}$, Tredano $\mathbf{M}$, et al. Interstitial lung disease in a baby with a de novo mutation in the SFTPC gene. Eur Respir J 2004;24:30-9.

24. Hamvas A, Nogee LM, White FV, et al. Progressive lung disease and surfactant dysfunction with a deletion in surfactant protein $\mathrm{C}$ gene. Am J Respir Cell Mol Biol 2004; 30:771-6.

25. Whitsett JA, Wert SE, Trapnell BC. Genetic disorders influencing lung formation and function at birth. Hum Mol Genet 2004;13:R207-15. 